\title{
Liquid crystal devices with continuous phase variation based on high-permittivity thin films
}

\author{
Oliver Willekens ${ }^{\mathrm{a}, \mathrm{b}}$, Kristiaan Neyts ${ }^{\mathrm{a}, \mathrm{b}}$, and Jeroen Beeckman ${ }^{\mathrm{a}, \mathrm{b}}$ \\ ${ }^{a}$ Ghent University, Sint-Pietersnieuwstraat 41, Dept. ELIS, Ghent, Belgium \\ ${ }^{\mathrm{b}}$ Center for Nano- and Biophotonics (NB-Photonics), Ghent University, Belgium
}

\begin{abstract}
Most liquid crystal devices use transparent conductive electrodes such as indium tin oxide (ITO) to apply a potential difference in order to achieve electro-optic switching. As an alternative, we study a device with narrow metallic electrodes in combination with dielectric layers with large dielectric permittivity. In this approach the applied voltage can be a continuous function of the lateral distance from the electrode line. Simulations for a one-dimensional beam-steering device show that the switching of the liquid crystal (LC) director depends indeed on the distance from the addressing electrodes and on the value of the relative permittivity. We show that in a device with electrodes spaced $60 \mathrm{\mu m}$ apart, the LC director halfway between the electrodes shows a considerable reorientation, when a dielectric layer with permittivity of $\epsilon_{r}=550$ is used, whereas no reorientation is observed for the uncoated reference sample at the same voltage. An added advantage is that the proposed configuration only contains dielectric materials, without resistive losses, which means that almost no heat is dissipated. This indicates that this technology could be used in low-power LC devices. The results show that using dielectric thin films with high relative permittivity in liquid crystal devices could form a cost-efficient and low-power alternative to many LC technologies where a gradient electric field is desirable.
\end{abstract}

Keywords: liquid crystal, dielectric layer, high-permittivity, gradient index

\section{INTRODUCTION}

Most displays nowadays are based on liquid crystals, a state of matter that resembles a liquid, because it flows, but exhibits some degree of order, just like solids. The most used property of liquid crystals is that the molecules reorient when an external field is applied. For practical applications, that is an electric field, but a magnetic field could also be used. ${ }^{1,2}$ For display applications, the electrodes need to be transparent. Indium tin oxide (ITO) has been a preferred material for this, because of its combination of good electrical conductivity, optical transparency and chemical resistance to moisture, but its main drawback is cost. Some alternatives exist, such as aluminium zinc oxide, carbon nanotubes and graphene, each with their own set of advantages and disadvantages. ${ }^{3,4}$

Not all liquid crystal applications require a uniform electric field. In beam-steering and beam-shaping applications, a smoothly varying optical path length is required. ${ }^{5}$ A spatial light modulator (SLM) is one such application. In an SLM, an array of closely spaced electrodes, each separately addressable, applies an inhomogeneous electric field over a liquid crystal, thereby generating a laterally varying optical path length. Fringe fields occur between two neighboring electrodes at different potentials relative to the common electrode and they distort the beam quality. An SLM is laboratory equipment however, and its enormous degree of freedom comes with complex technology and hence, cost. Some liquid crystal beam-steering applications for the consumermarket are on the horizon: in autostereoscopic 3D displays liquid crystal lenses are used to provide the 3D effect. ${ }^{6,7}$ They can be made with lenticulars ${ }^{8-10}$ or hole-patterned arrays, ${ }^{11}$ but the best results are achieved with multi-electrode designs, similar to the SLM, but with larger spacing between the electrodes to reduce the complexity. The subregions of the liquid crystal that are not sandwiched between two electrodes do not switch very well, so researchers have proposed some techniques to overcome this: Li et al. proposed a series of concentric electrodes with inter-electrode resistors that are interconnected via small resistive patches. ${ }^{12}$ De Smet et al. used

O. Willekens: E-mail: oliver.willekens@elis.ugent.be, Telephone: +329264 8951

J. Beeckman: E-mail: jeroen.beeckman@elis.ugent.be, Telephone: +3292643376

Emerging Liquid Crystal Technologies XI, edited by Liang-Chy Chien, Dick J. Broer, Hirotsugu Kikuchi, Nelson V. Tabiryan, Proc. of SPIE Vol. 9769, 97690P

(C) 2016 SPIE · CCC code: 0277-786X/16/\$18 · doi: 10.1117/12.2209326 
poly(3,4-ethylenedioxythiophene):poly(styrenesulfonate), a conductive polymer also knows as PEDOT:PSS, to add flexibility to their lens design. ${ }^{13}$ In these cases, conductors that physically connect to the addressing electrodes have been used and these systems therefor draw a non-negligible current. Li et al. proposed floating electrodes to "effectively increase the phase sampling". ${ }^{14}$ Theirs is an example of a configuration that does not draw resistive current, which could be an important design consideration when fabricating lenses for e.g. mobile devices. In this manuscript, we study an alternative to generate a smooth, continuous phase profile for gradient index applications by means of numerical simulation: we show that by coating the addressing electrodes with a dielectric layer with high permittivity the optical path length profile varies smoothly in the lateral direction.

\section{MESH DESCRIPTION}

We have simulated the director profile of the uniaxially birefringent liquid crystal mixture E7 within the twodimensional structure that is shown in Fig. 1a. It resembles a liquid crystal cell where the LC is sandwiched between two glass substrates, one of which has a uniform conductive coating (the "common electrode"), the other having a finger electrode pattern on top of it. To study the effect of a dielectric layer over the finger electrodes, we have compared simulations of this geometry without and with a dielectric layer present. The simulation program is based on the Finite Element Method and computes the Q-tensor in each of the nodes of the mesh shown in Fig. 1b. ${ }^{15}$

The geometry to be meshed, shown in Fig. 1a, consists of 4 domains: from the top to the bottom these represent the liquid crystal, the dielectric layer, a thin buffer layer and a bottom glass substrate. The reason a $2^{\text {nd }}$ dielectric layer is present, is one of convenience and forethought: in an upcoming paper, we have fabricated the proposed geometry and this $2^{\text {nd }}$ dielectric layer is physically required to ensure the quality of the highpermittivity layer on top. The thickness of this layer is almost negligible compared to the $2^{\text {nd }}$ layer, as shown in Fig. 1b, but its relative dielectric constant is low $\left(\epsilon_{2, r}<20\right)$. Our interest goes out to the influence of the $1^{\text {st }}$ dielectric layer, so all other references to "the dielectric layer" in this manuscript are about the $1^{\text {st }}$ layer, unless explicitly mentioned otherwise.

The mesh has periodic boundary conditions on the left and right sides and a Neumann boundary condition on the bottom. An electrode on the top, spanning the entire width of the liquid crystal layer, and a smaller width electrode underneath the liquid crystal layer are responsible for switching the LC. Because the common electrode (Dirichlet boundary condition) extends over the entire LC domain, we do not need to model the layer on top of it, which in real life applications would usually be a glass or silicon substrate.

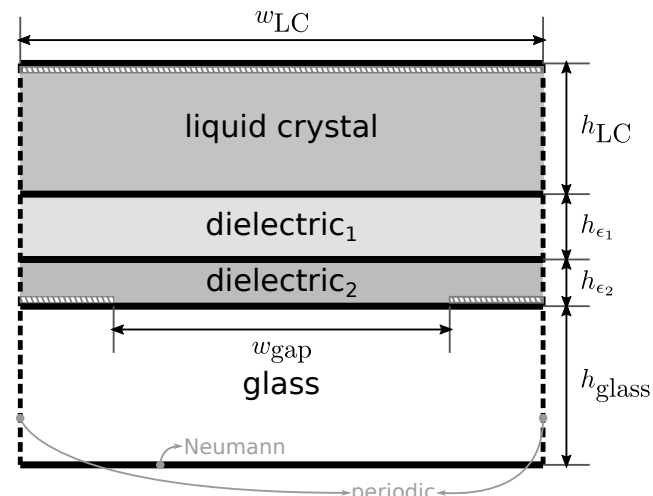

(a)

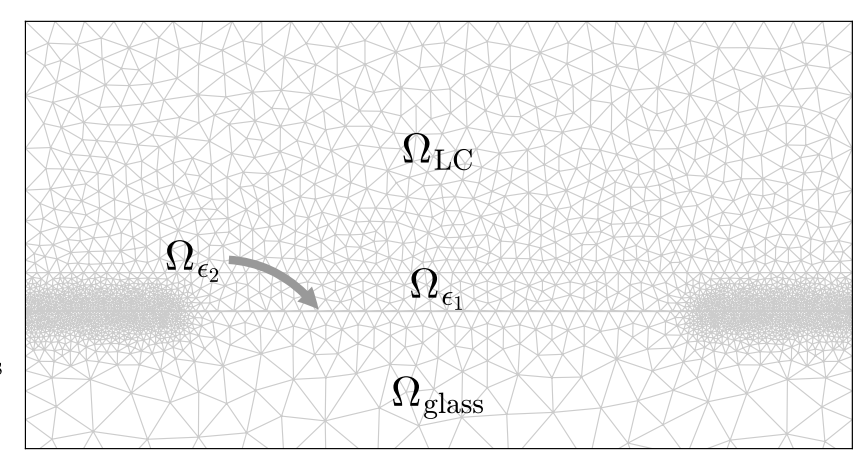

(b)

Figure 1: Schematic representation of the geometry used in the simulations. (a) The geometry is fully described by 6 parameters, denoted in the figure. The thickness of the electrodes (hatched regions) is exaggerated to show their relative position. (b) A close-up view of the generated mesh in the vicinity of the addressing electrodes (the densely meshed regions). Note that the thickness of the $2^{\text {nd }}$ dielectric is negligible. 


\subsection{Determination of an Upper Bound on the Glass Thickness}

It is tempting to reduce the height of the glass layer to a minimum, in order to reduce the size of the computational domain, as we are not interested in the electric field distribution within the glass domain. However, some of the electric field lines originating at an addressing electrode will extend into the glass region before ending up at the common electrode. Yet, modelling the lower domain to realistic scales compared to the size of the liquid crystal layer (200:1) would require far too much computation time for each of the characteristics we have studied. We therefor determined an upper bound on the height of the glass layer by studying the absolute error of the scalar potential, which we defined as follows:

$$
\operatorname{err}_{i}:=\left|V_{h_{i}}(\vec{r})-V_{h_{1000}}(\vec{r})\right| \quad \text { with } \quad \vec{r} \in \Omega_{\mathrm{LC}}
$$

where $V_{h_{1000}}(\vec{r})$ is the scalar potential when the height of the glass layer is $1000 \mu \mathrm{m}$ and $V_{h_{i}}(\vec{r})$ the scalar potential in the mesh with a different height for the glass layer. Intuitively one might expect the error to be largest furthest away from the electrodes. Figure 2 shows that this is indeed the case. It is a visual representation of equation 1 , for the case when no dielectric layer is present. The electrodes, not technically part of the LC domain, have been added as an overlay, with largely exaggerated height: in typical LC devices, they are usually less than $0.2 \mu \mathrm{m}$ thick.

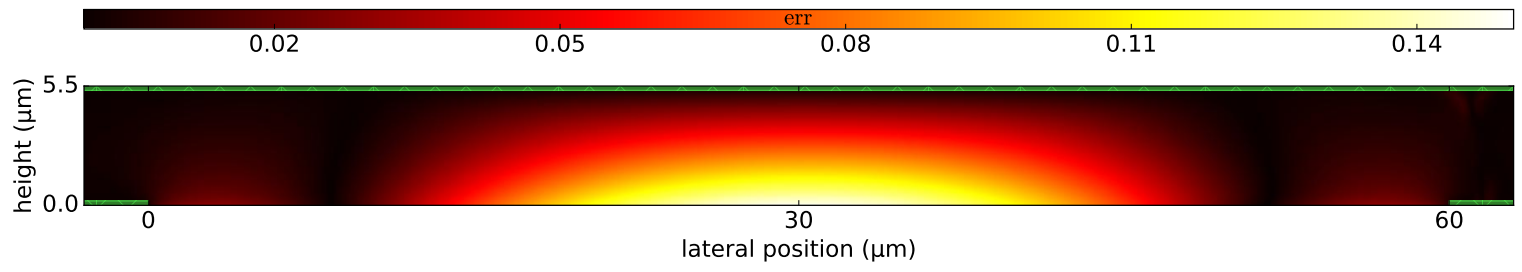

Figure 2: Visualization of the absolute error (defined in equation 1) when the height of the glass layer is $10 \mu \mathrm{m}$ and the potential difference is $5 \mathrm{~V}$. Dielectric layers are not present.

Figure 3 shows the maximum absolute error in the LC domain as a function of the height of the glass substrate for both the reference case and the case where the dielectric layer $\left(\epsilon_{r}=550\right)$ is present. It shows that the error initially drops fast, almost logarithmically for the case when no dielectric is present, with increasing height of the glass layer. It also indicates that the absolute error is reduced to less than $0.025 \mathrm{~V}$ in both cases, once the height of the glass layer is more than about $20 \mu \mathrm{m}$. This absolute error, compared to the potential difference between the common electrode and the finger electrode $(5 \mathrm{~V})$, is an error of less than $0.5 \%$.

A relative error, as defined by:

$$
\operatorname{err}_{r, i}:=\left|\frac{V_{h_{i}}(\vec{r})-V_{h_{1000}}(\vec{r})}{V_{h_{1000}}(\vec{r})}\right| \quad \text { with } \quad \vec{r} \in \Omega_{\mathrm{LC}},
$$

would not lead to a meaningful conclusion, because there are positions $\vec{r}$ for which $V_{h_{1000}}(\vec{r})$ is very small. Taking an offset (DC bias) into account for the potential of the electrodes would solve the problem, but creates a new one: the relative error would no longer be unique.

Care should be taken when generalizing the result of Fig. 3 however: we simulated the dynamics of a $5.5 \mu \mathrm{m}$ thick layer of liquid crystal with an electrode spacing of $w_{\text {gap }}=60 \mu \mathrm{m}$, the largest gap size we took into consideration. When these geometric parameters change, the absolute error will be different. Changes in the physical constants of the problem will have similar effects. In this manuscript we focus on a specific case, for a soon to be published paper, where the liquid crystal layer thickness is $5.5 \mu \mathrm{m}$ and the material constants of the LC are those of the nematic LC mixture E7 [16, p.22]. All of the cases we have studied so far have an upper limit of the electrode spacing of $w_{\text {gap }}=60 \mu \mathrm{m}$. The absolute errors will be smaller when the electrodes are more closely spaced. For our simulations, we follow a general guideline that the glass layer thickness should be about the size of the interelectrode spacing. 


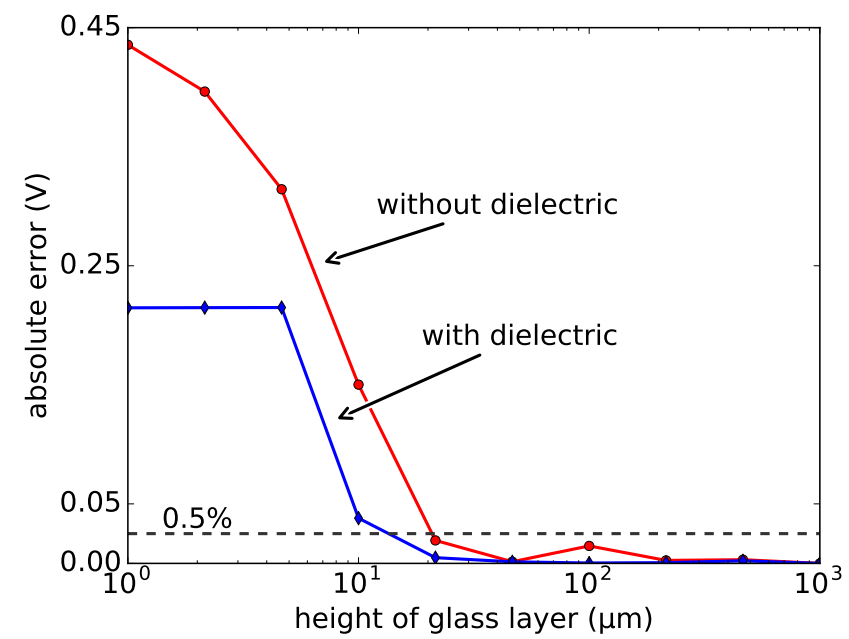

Figure 3: The absolute error in the potential distribution in the LC domain decreases rapidly with increasing height of the glass layer, but there is very little benefit to be found once the glass layer thickness is about $20 \mu \mathrm{m}$. For these results, the dielectric is assumed to have a high permittivity of $\epsilon_{r}=550$.

\section{AVERAGE REFRACTIVE INDEX}

Many electro-optic applications involving liquid crystals only consider two states: a voltage-ON state, where the liquid crystal is fully switched, and a voltage-OFF state, where the liquid crystal is in its initial state, which is largely determined by the boundary conditions imposed by the alignment layers, such as anchoring strength and pretilt. In both cases the physics can usually be simplified by considering the average refractive index experienced by light propagating through the LC layer:

$$
\langle n\rangle=\frac{1}{d} \int_{0}^{d} n_{\mathrm{eff}}(z) d z
$$

where $d$ is the thickness of the LC layer and $n_{\mathrm{eff}}(z)$ is the effective refractive index at a height of $z$ within the layer. The effective refractive index is a function of the tilt and twist of the LC director, the unit vector representing the average direction of the liquid crystal molecules. When there is no twist, the difference between the voltage-ON and voltage-OFF state is determined only by the tilt of the director and $n_{\text {eff }}(z)$ is given by ${ }^{17}$

$$
n_{\mathrm{eff}}=\left[\frac{\cos ^{2} \theta}{n_{e}^{2}}+\frac{\sin ^{2} \theta}{n_{o}^{2}}\right]^{-1 / 2}
$$

with $n_{e}$ and $n_{o}$ the material constants representing the extraordinary and ordinary refractive index of the LC respectively. The tilt of the director, $\theta$, is the angle between the director and its projection on the xy-plane, which is perpendicular to the propagation direction of light.

\subsection{Influence of the Permittivity}

Shown in Fig. 4 are the simulated director profiles of the LC cell geometry shown in Fig. 1a, for two cases: in Fig. 4a the dielectric layer has a relative permittivity of $\epsilon_{r}=20$, in Fig. $4 \mathrm{a} \epsilon_{r}=550$. A pretilt of $2^{\circ}$ (counterclockwise) was imposed, with strong anchoring. In both cases, an electric potential difference of $3 \mathrm{~V}$ was applied between the common and addressing electrodes. Between the addressing electrodes and the common, this potential is sufficient to switch the liquid crystal from its planar configuration to a direction almost perfectly perpendicular to the common electrode; the director tilt is close to $90^{\circ}$. As the distance to the addressing electrodes increases, the director tilt decreases. This change occurs faster when the permittivity of the dielectric layer over the addressing electrodes is lower (Fig. 4a). One region in Fig. 4a stands out: close to the electrode on the right side, the director is tilted in a clockwise fashion because of the electric field emanating from this electrode. This 
clockwise reorientation opposes the pretilt imposed by the alignment layer $\left(\left.\theta\right|_{z=0, d}=2^{\circ}\right)$ and a domain wall is formed. A similar profile is observed in absence of the dielectric layer. Fig. $4 \mathrm{~b}$ indicates that a value of 550 for the permittivity of the dielectric layer prevents the formation of such a domain wall and the director profile assumes a counterclockwise tilt over the entire domain.

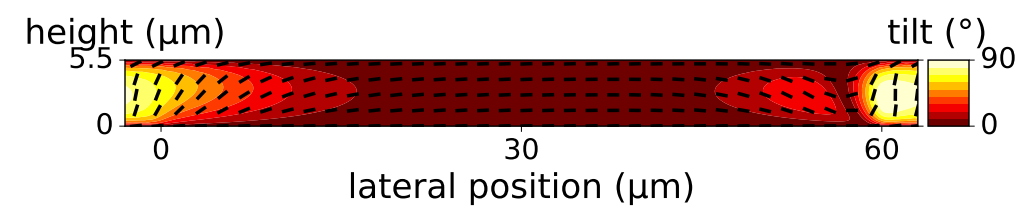

(a)

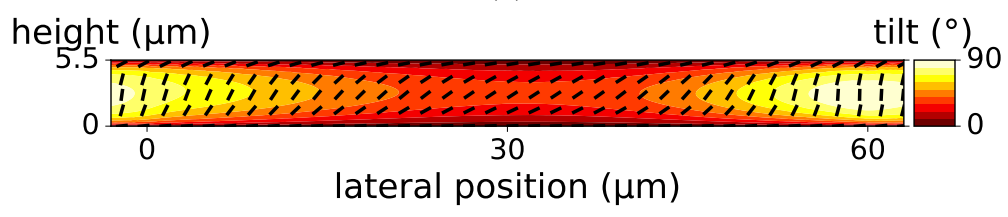

(b)

Figure 4: The director profile of the liquid crystal domain when a potential difference of $3 \mathrm{~V}$ is applied between the addressing electrode and the common. The addressing electrodes are spaced $60 \mu \mathrm{m}$ apart. In (a) the permittivity of the dielectric layer over the addressing electrodes is $\epsilon_{r}=20$. In (b) $\epsilon_{r}=550$. A domain wall can be seen to the left of the electrode on the right in (a).

Equation 3 can be computed numerically from these simulation results. It is shown in Fig. 5, as a function of the lateral distance, for different values of the dielectric constant of the dielectric layer $\epsilon_{1}$, when the applied potential is $3 \mathrm{~V}$.

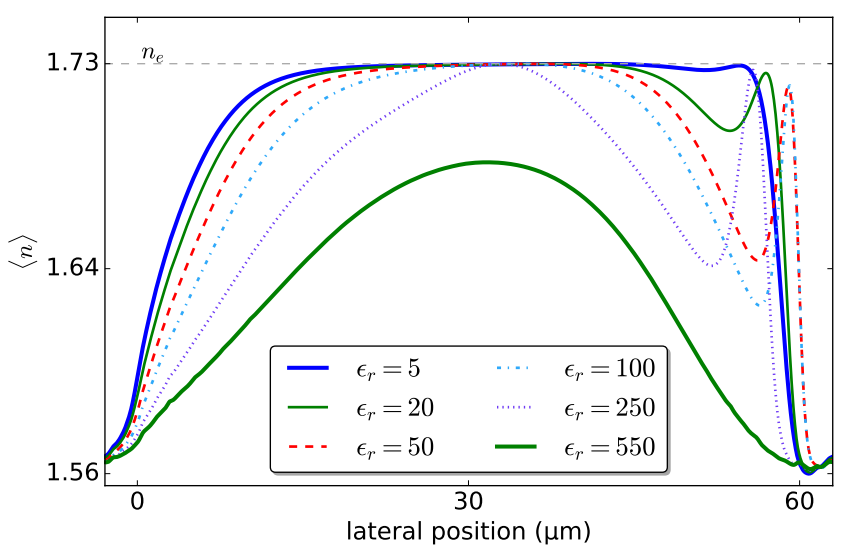

Figure 5: The average refractive index for monochromatic light $(\lambda=633 \mathrm{~nm})$ linearly polarized along the initial LC alignment direction and travelling parallel to the interface normal of the cell shown in Fig. 1a. As the permittivity of the dielectric layer increases, the liquid crystal further away from the addressing electrodes starts to reorient.

We begin the discussion of Fig. 5 by noting that the average refractive index sensed over the addressing electrode (i.e. $x<0 \mu \mathrm{m}$ or $x>60 \mu \mathrm{m}$ ) is largely independent of the dielectric constant of the dielectric layer, and always close to a value of 1.56, which is to be expected: as shown in Fig. 4, the director profile above the addressing electrode will be mostly $90^{\circ}$ tilted, such that light experiences mostly $n_{o}$. As the distance to the electrode to the left increases, $\langle n\rangle$ increases and saturates at a value of 1.73 , which is the extraordinary refractive index of E7 at the simulated wavelength of $633 \mathrm{~nm}$. This indicates that the liquid crystal has not switched at the position where $\langle n\rangle \approx n_{e}$. The position where the saturation starts is moved further away from the electrode as the permittivity of the dielectric layer increases. For $\epsilon_{r}=550$ there is no saturation, which indicates that the 
LC has been brought to threshold over the entire electrode gap. We also observe, close to the left edge of the electrode, an oscillation in $\langle n\rangle$. The explanation for this is found in our earlier analysis of the director profiles: in the domain wall of Fig 4a, the director tilt varies rapidly between two angles with opposite sign and passes $0^{\circ}$ in between. Where it is $0^{\circ}$, linearly polarized light will experience the extraordinary refractive index of the liquid crystal.

\subsection{Influence of the Electrode Spacing}

The interelectrode spacing will also influence the average refractive index. As the interelectrode spacing decreases, the optical path length becomes more easily controllable. When a specific spacing is imposed, it could be possible to still get reasonable control over the optical path length, by tuning the permittivity of the dielectric layer (as shown in the previous section) or by tuning the thickness of the dielectric layer.

Figure 6 shows the average refractive index for monochromatic light $(\lambda=633 \mathrm{~nm})$ experienced by light travelling along the interface normal of Fig. 1a, when the potential difference between the common and the addressing electrode is $2 \mathrm{~V}$, for a range of interelectrode spacings. To allow easy comparison, the lateral position has been normalized, because the mesh width $\left(w_{\mathrm{LC}}\right.$ in Fig. 1a) changes with the interelectrode spacing.

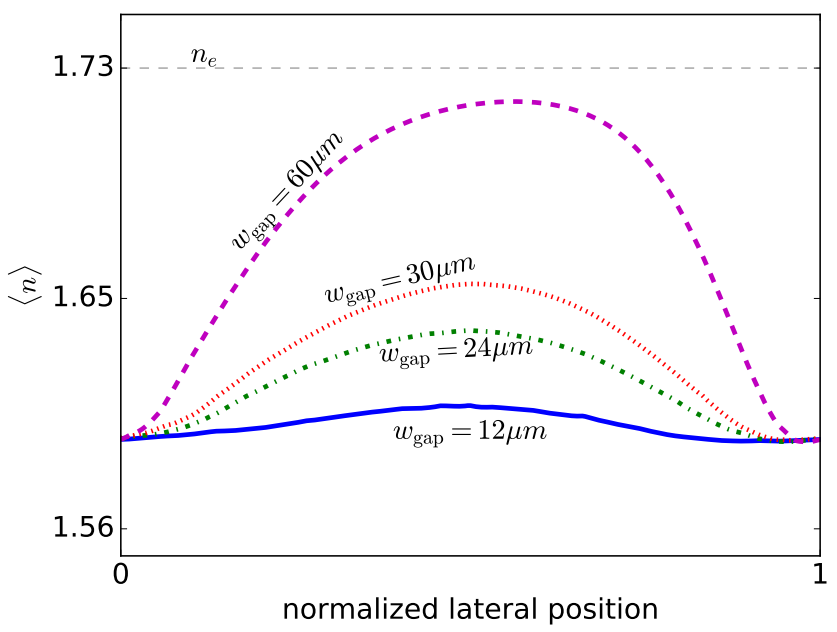

Figure 6: The average refractive index for monochromatic light $(\lambda=633 \mathrm{~nm}$ ) travelling parallel to the interface normal of the cell shown in Fig. 1a. The linearizing effect of the dielectric layer $\left(\epsilon_{r}=550\right.$, $\left.h_{\epsilon_{1}}=0.84 \mu \mathrm{m}\right)$ is limited by the interelectrode spacing.

Here too, the refractive index experienced just above the addressing electrodes is nearly independent of the interelectrode spacing and for the same reason as above. A nearly constant index is obtained when the interelectrode spacing is $12 \mu \mathrm{m}$ with $\epsilon_{r}=550$, a value that can be reached with certain ferroelectric materials such as barium titanate $\left(\mathrm{BaTiO}_{3}\right)$ and lead zirconate titanate $(\mathrm{PZT})$, which both have a perovskite structure. ${ }^{18}$

\section{CONCLUSIONS}

We have shown that a dielectric layer with high permittivity can be used to create a capacitive bridge between neighboring addressing electrodes in liquid crystal applications. The bridge extends the electric field laterally from the electrodes and can thus be used to switch a liquid crystal farther away from the usual influence field of the electrodes. The dielectric layer thickness can be an important design parameter, given the interelectrode spacing. We have also shown that a dielectric layer with $\epsilon_{r}=550$, a value that can be reached by certain ferroelectric materials such as PZT, can be used to prevent the appearance of domain walls.

\section{ACKNOWLEDGMENTS}

The authors acknowledge financial support from the "Agentschap voor Innovatie door Wetenschap en Technologie" IWT through the SBO project SECONDOS, IWT-nr 120019, 2013-2016, as well as the Interuniversity 
Attraction Poles program of the Belgian Science Policy Office, under grant IAP P7-35 (Photonics@be). Our thanks also go to Filip Strubbe and Filip Beunis, our colleagues, for fruitful discussions.

\section{REFERENCES}

[1] Challa, P. K., Curtiss, O., Williams, J., Twieg, R., Toth, J., McGill, S., Jákli, A., Gleeson, J., and Sprunt, S. N., "Light scattering from liquid crystal director fluctuations in steady magnetic fields up to 25 tesla," Physical Review E 86(1), 011708 (2012).

[2] Wang, M., He, L., Zorba, S., and Yin, Y., "Magnetically actuated liquid crystals," Nano Letters 14(7), 3966-3971 (2014).

[3] Minami, T., "Present status of transparent conducting oxide thin-film development for indium-tin-oxide (ito) substitutes," Thin Solid Films 516(17), 5822-5828 (2008).

[4] Blake, P., Brimicombe, P. D., Nair, R. R., Booth, T. J., Jiang, D., Schedin, F., Ponomarenko, L. A., Morozov, S. V., Gleeson, H. F., Hill, E. W., Geim, A. K., and Novoselov, K. S., "Graphene-based liquid crystal device," Nano Letters 8(6), 1704-1708 (2008).

[5] McManamon, P. F., Bos, P. J., Escuti, M. J., Heikenfeld, J., Serati, S., Xie, H., Watson, E., et al., "A review of phased array steering for narrow-band electrooptical systems," Proceedings of the IEEE 97(6), 1078-1096 (2009).

[6] Huang, Y.-P., Chen, C.-W., and Huang, Y.-C., "Superzone fresnel liquid crystal lens for temporal scanning auto-stereoscopic display," Journal of Display Technology 8(11), 650-655 (2012).

[7] Huang, Y.-P., Liao, L.-Y., and Chen, C.-W., "2-d/3-d switchable autostereoscopic display with multielectrically driven liquid-crystal (med-lc) lenses," Journal of the Society for Information Display 18(9), 642-646 (2010).

[8] Ren, H., Fan, Y.-H., Gauza, S., and Wu, S.-T., "Tunable-focus flat liquid crystal spherical lens," Applied Physics Letters 84(23), 4789-4791 (2004).

[9] Li, Y. and Wu, S.-T., "Polarization independent adaptive microlens with a blue-phase liquid crystal," Opt. Express 19, 8045-8050 (Apr 2011).

[10] Lin, S.-H., Huang, L.-S., Lin, C.-H., and Kuo, C.-T., "Polarization-independent and fast tunable microlens array based on blue phase liquid crystals," Opt. Express 22, 925-930 (Jan 2014).

[11] Nose, T. and Sato, S., "A liquid crystal microlens obtained with a non-uniform electric field," Liquid Crystals 5(5), 1425-1433 (1989).

[12] Li, L., Bryant, D., and Bos, P. J., "Liquid crystal lens with concentric electrodes and inter-electrode resistors," Liquid Crystals Reviews 2(2), 130-154 (2014).

[13] De Smet, J., Avci, A., Joshi, P., Cuypers, D., and De Smet, H., "P-1591: Late-news poster: A liquid crystal based contact lens display using pedot: Pss and obliquely evaporated sio2," SID Symposium Digest of Technical Papers 43(1), 1375-1378 (2012).

[14] Li, L., Bryant, D., Heugten, T. V., and Bos, P. J., "Near-diffraction-limited and low-haze electro-optical tunable liquid crystal lens with floating electrodes," Opt. Express 21, 8371-8381 (Apr 2013).

[15] James, R., Willman, E., Fernández, F. A., and Day, S. E., "Finite-element modeling of liquid-crystal hydrodynamics with a variable degree of order," IEEE Transactions on Electron Devices 53(7), 1575-1582 (2006).

[16] Yeh, P. and Gu, C., [Optics of liquid crystal displays], John Wiley \& Sons (2010).

[17] Si, G., Zhao, Y., Leong, E. S. P., and Liu, Y. J., "Liquid-crystal-enabled active plasmonics: a review," Materials 7(2), 1296-1317 (2014).

[18] George, J. P., Smet, P. F., Botterman, J., Bliznuk, V., Woestenborghs, W., Van Thourhout, D., Neyts, K., and Beeckman, J., "Lanthanide-assisted deposition of strongly electro-optic pzt thin films on silicon: Toward integrated active nanophotonic devices," ACS Applied Materials 85 Interfaces 7(24), 13350-13359 (2015). 